\title{
Analysis and Comparison of Distance Vector, DSDV and AODV Protocol of MANET
}

\author{
Prof. B.N. Jagdale ${ }^{1}$, Prof. Pragati Patil ${ }^{2}$, Prof. P. Lahane ${ }^{3}$, Prof. D. Javale ${ }^{4}$ \\ Department of Computer Engineering, MIT College of Engineering, Pune, India \\ pragatimitcoeegmail.com, pragatimitegmail.com
}

\begin{abstract}
In MANET, mobile nodes communicate with each other using wireless channels, without any existing infrastructure. Transmission of all kinds of data will takes place with the help of multiple hops across the network, because the transmission as well as reception range of wireless network is limited. Therefore, routing protocol plays a vital role to facilitate communication within network and is also used to discover proper route between nodes. AODV, DSDV, DSR etc. are the most popular routing protocol for MANET which are used to established correct and efficient path between nodes.

This paper examines working of two routing protocols for MANET- the Destination Sequenced Distance Vector (DSDV) which is a proactive protocol depending on routing tables which are maintained at each node and Ad hoc On-Demand Distance Vector routing (AODV) which find route to a destination on demand, whenever communication is needed and their comparison based on certain parameters.
\end{abstract}

\section{Keywords}

$A O D V, D S D V$, Distant Vector, MANET

\section{Introduction}

In MANET, network structure changes dynamically, because of mobile nature of the nodes. That is the reason MANET are said to be self organizing and configuring wireless network. For the communication purpose, nodes make use of random access wireless channel to forward the data. The nodes in MANET act as hosts as well as routers that routes data to and from others nodes in network. If the destination node is out of range from the source node who is transmitting the data, then a routing procedure is always needed to find out the appropriate path between source and destination so that the forwarded packet reach to its destination.[3]

Following are the problems with routing in Mobile Ad-hoc Networks:

1. Asymmetric links

The links in MANET are asymmetric because of the mobile nature of nodes and their continuously changing position within network. But wired networks are always fixed therefore can rely on the symmetric links..

\section{Routing Overhead}

As nodes are mobile, their positions are continuously changes therefore, in routing table some fake path/routes are generated in which leads to routing overhead.

3. Interference 
International Journal of Distributed and Parallel Systems (IJDPS) Vol.3, No.2, March 2012

This is the major problem with mobile ad-hoc networks as links come and go depending on the transmission characteristics, one transmission might interfere with another one and node might overhear transmissions of other nodes and can corrupt the total transmission.

\section{Dynamic Topology}

Topology of a network can't be constant. The nature of the mobile nodes is unpredictable, they might move or stable or characteristics of communication media might change [3].

\section{Classification of Routing Protocol in MANET's}

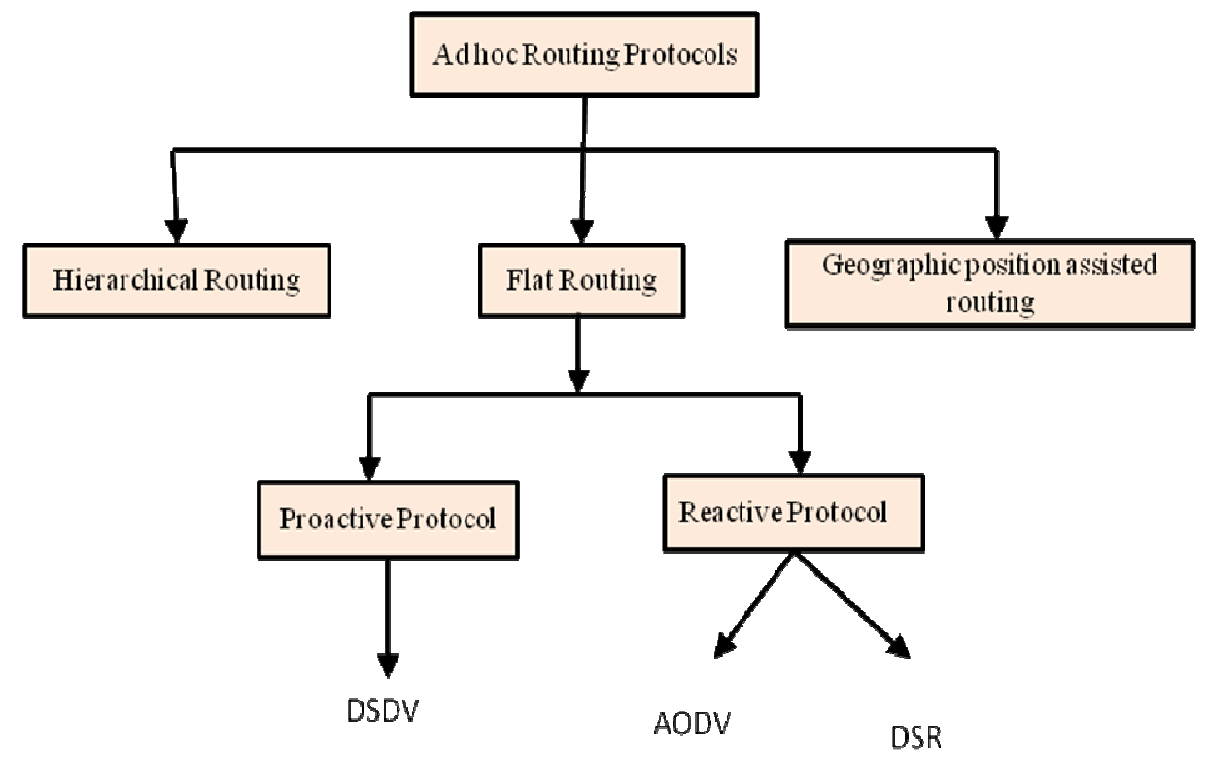

Figure 1. Classification of Routing Protocols In Mobile Ad-hoc Networks

From the above classification, the paper mainly focused on Distance Vector, DSDV, \& AODV protocol and their analysis.

\section{Distance-Vector Protocol}

Distant vector protocol is also known as Distributed Bellman-Ford or RIP (Routing Information Protocol). Every node maintains a routing table and it contains all available destinations details, the next node to reach to destination, the number of hops to reach the destination. To maintain topology in a network nodes periodically send table to all neighbors. Following figure depicts the tables maintained by three nodes (A,B \& C).

By using the distance vector protocols, each router over the internetwork send the neighbouring routers, the information about destination that it knows how to reach. Moreover to say the routers sends two pieces of information first, the router tells, how far it thinks the destination is and secondly, it tells in what direction (vector) to use to get to the destination. When the router receives the information from the others, it could then develop a table of destination addresses, distances and associated neighbouring routers, and from this table then select the shortest route to the destination. Using a distance vector protocol, the router simply forwards the packet to the neighbouring host (or destination) with the available shortest path in the routing table and assumes that the receiving router will know how to forward the packet beyond that point. The best example for this is the routing information protocol (RIP).[3] 
International Journal of Distributed and Parallel Systems (IJDPS) Vol.3, No.2, March 2012

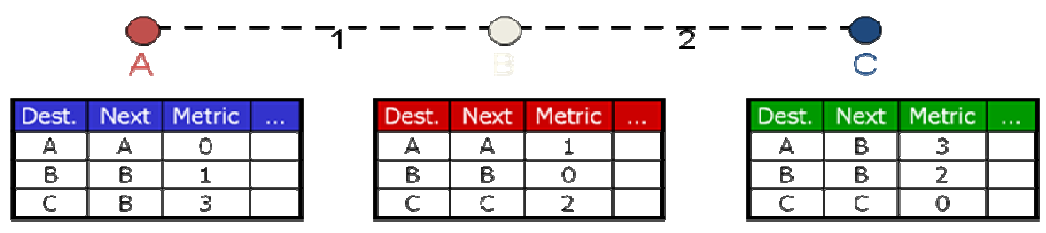

Figure 2. Distant Vector Table

The below figure represents that, how node updates their routing information by maintaining a routing table.

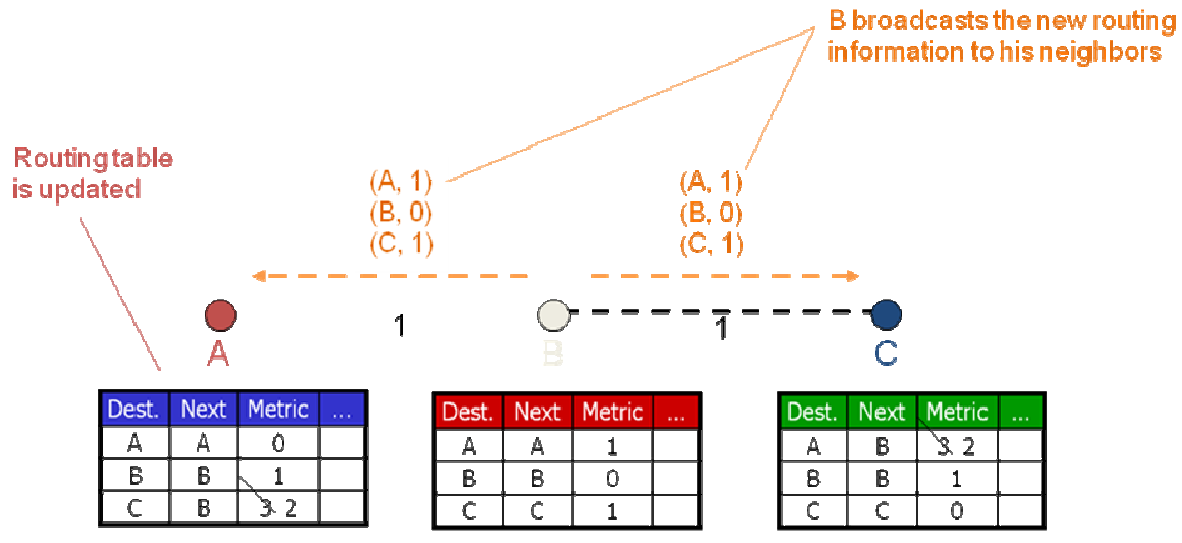

Figure 3. Updation in routing table

As and when the nodes broadcast new routing information in a network, nodes will update their routing tables as shown in figure 3 .

\subsection{Limitations of Distant-Vector protocol}

\subsubsection{Distance Vector (Broken Link)}

The following diagrams shows a problem in Distant vector when any link breaks down. Four nodes A,B,C, \&D are shown in the figure and the link between C \& D s broken down.

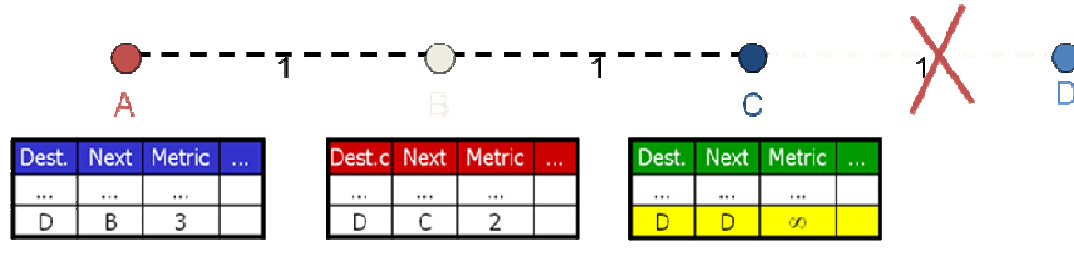

Figure 4. Broken link in distant vector 
3.1.2. Distance Vector (Loops)

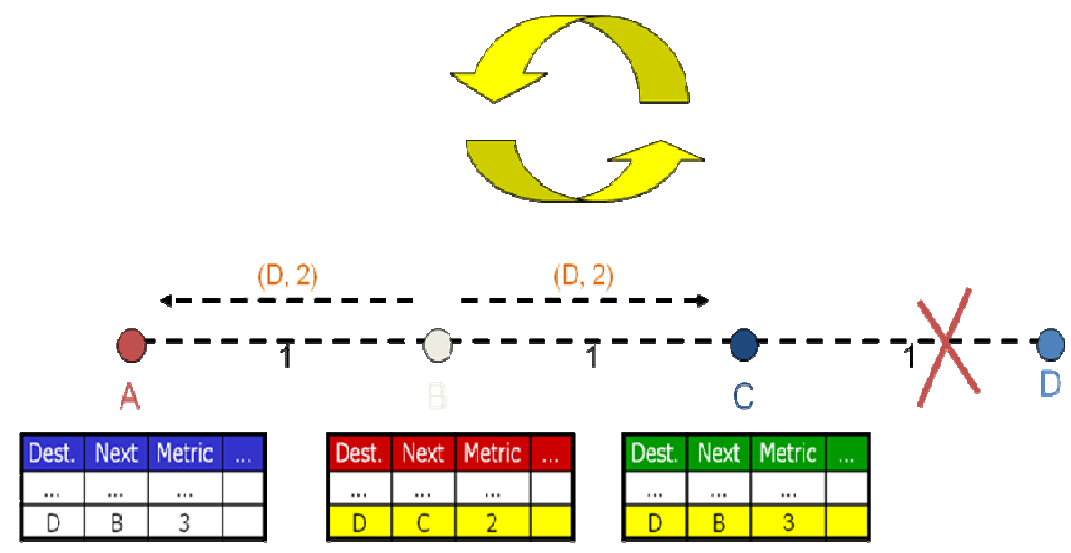

Figure 5. Loops protocol in distant vector

DV not suited for ad-hoc networks because of Loops \& Count to Infinity. So, the solution over DV is DSDV Protocol

\section{Destination sequenced Distance Vector (DSDV) Protocol}

DSDV protocol is a proactive routing protocol which is a modification of conventional Bellman-Ford routing algorithm. In this protocol each nodes maintains routing table. This routing information must be periodically updated. With the help of routing information nodes can transmit data to other node in a network. The fields of routing table are as following: destination, next, metric, sequence number, installs time, stable data etc.. Sequence numbers are basically originated from destination itself which ensures loop freeness. Install time are used to delete fake entries from table. Stable data is basically a pointer to a table holding information on how stable a route is and also used to damp fluctuations in network.

\subsection{Protocol Activities}

In MANET, each node maintains and update the routing table, which are needed for the transmission of data. The table contains all the available destination and the number of hops to reach it. To maintain consistency, each node in a network transmit packet and update it periodically. The packets are being broadcasted to find out which stations are in vicinity and how many number of hops required to reach the destination. Packets may be transmitted containing the layer 2 or layer 3 address.

This protocol ensures that each node in a network continuously advertises to each of their neighbour, so that the updation of routing table has be known to all nodes and they might be in a position to find out shortest path to reach destination. Therefore, even if there is no direct link between the nodes, can exchange data. The data broadcast by each node contain new sequence number and the following information for each new route:

- The destination address

- The number of hops required to reach the destination

- The new sequence number, originally stamped by the destination

When destination node received data packet, it compares the sequence number of received packet with the available entry list. If the sequence number is larger then it will update routing table information with new sequence number. If the comparison of sequence number is found to 
International Journal of Distributed and Parallel Systems (IJDPS) Vol.3, No.2, March 2012

be same, then it looks for the metric entry in a table and if the number of hops is less than the previous entry the new information is updated. The metric is increased by 1 and sequence number is increased by 2 when the nodes information is updated [5][1].

\subsection{Routing table of DSDV}

Consider the figure which has 8 hosts in the network. We will have a look at the changes to the MH4 routing table with reference to the movements of MH1. Initially, all the nodes advertise their routing information to all the nodes in the network and hence the routing table at MH4 initially looks like [5].

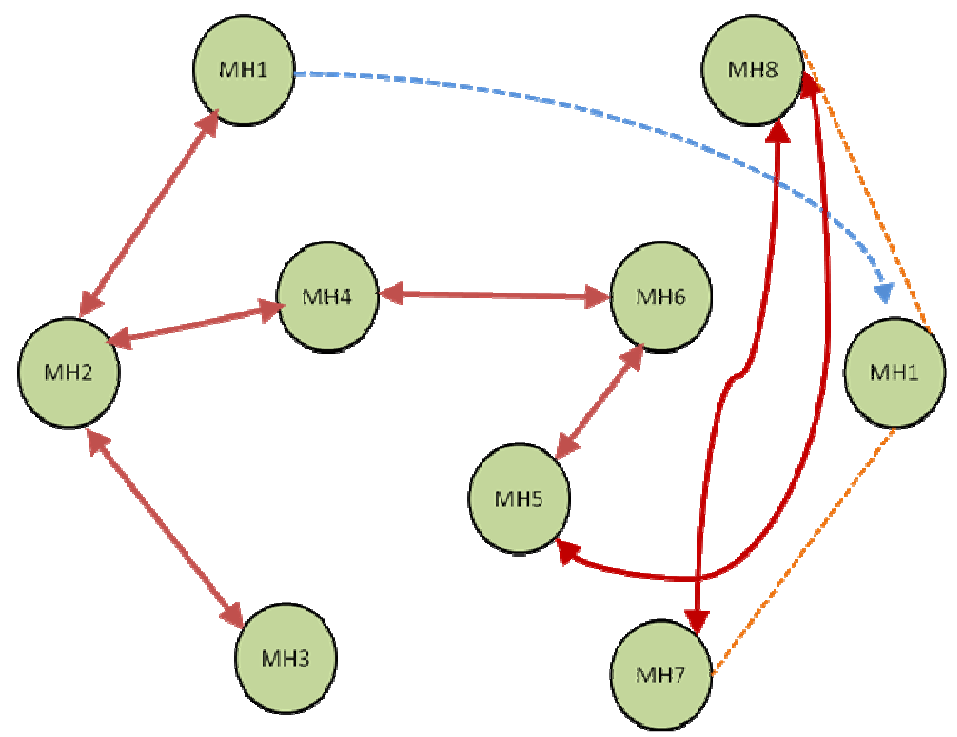

Figure 6. Movement of Mobile host in Adhoc Networks.

Routing table of MH4 And the forwarding table at the MH4 would look like this. But, when the host MH1 moves its location as shown in the figure. Nearer to MH7 and MH8 then, the link between $\mathrm{MH} 2$ and $\mathrm{MH} 1$ will be broken resulting in the assignment of infinity metric at $\mathrm{MH} 2$ for $\mathrm{MH} 1$ and the sequence number will be changed to odd number in the routing table at MH2.

Table 1. Forwarding table at MH4

\begin{tabular}{|l|l|l|l|l|l|}
\hline Destination & Next Hop & Metric & $\begin{array}{l}\text { Sequence } \\
\text { Number }\end{array}$ & Install & Stable Data \\
\hline MH1 & MH2 & 2 & S406_MH1 & T001_MH4 & Ptr1_MH1 \\
\hline MH2 & MH2 & 1 & S128_MH2 & T001_MH4 & Ptr1_MH2 \\
\hline MH3 & MH2 & 2 & S564_MH3 & T001_MH4 & Ptr1_MH3 \\
\hline
\end{tabular}


International Journal of Distributed and Parallel Systems (IJDPS) Vol.3, No.2, March 2012

\begin{tabular}{|l|l|l|l|l|l|}
\hline MH4 & MH4 & 0 & S710_MH4 & T001_MH4 & Ptr1_MH4 \\
\hline MH5 & MH6 & 2 & S392_MH5 & T002_MH4 & Ptr1_MH5 \\
\hline MH6 & MH6 & 1 & S076_MH6 & T001_MH4 & Ptr1_MH6 \\
\hline MH7 & MH6 & 2 & S128_MH7 & T002_MH4 & Ptr1_MH7 \\
\hline MH8 & MH6 & 3 & S050_MH8 & T002_MH4 & Ptr1_MH8 \\
\hline
\end{tabular}

And the forwarding table at the MH4 would look like this

Table 2. Forwarding table at MH4

\begin{tabular}{|c|c|c|}
\hline Destination & Metric & Sequence Number \\
\hline MH1 & 2 & S406_MH1 \\
\hline MH2 & 1 & S128_MH2 \\
\hline MH3 & 2 & S564_MH3 \\
\hline MH4 & 0 & S710_MH4 \\
\hline MH5 & 2 & S392_MH5 \\
\hline MH6 & 1 & S076_MH6 \\
\hline MH7 & 2 & S128_MH7 \\
\hline MH8 & 3 & S050_MH8 \\
\hline
\end{tabular}

But, when the host MH1 moves its location as shown in the figure 6 nearer to MH7 and MH8 then, the link between $\mathrm{MH} 2$ and $\mathrm{MH} 1$ will be broken resulting in the assignment of infinity metric at $\mathrm{MH} 2$ for $\mathrm{MH} 1$ and the sequence number will be changed to odd number in the routing table at MH2. MH2 will update this information to its neighbour hosts. Since, there is a new neighbour host for MH7 and MH8; they update their information in the routing tables and they broadcast. Now, MH4 will receive its updated information from MH6 where MH6 will receive two information packets from different neighbours to reach MH1 with same sequence number, but different metric. The selection of the route will depend on less hop count when the sequence number is the same. Now the routing table will look like

Table 3. Routing table after MH1 movement

\begin{tabular}{|l|l|l|l|l|l|}
\hline Destination & Next Hop & Metric & $\begin{array}{l}\text { Sequence } \\
\text { Number }\end{array}$ & Install & Stable Data \\
\hline MH1 & MH6 & 3 & S516_MH1 & T001_MH4 & Ptr1_MH1 \\
\hline MH2 & MH2 & 1 & S238_MH2 & T001_MH4 & Ptr1_MH2 \\
\hline
\end{tabular}


International Journal of Distributed and Parallel Systems (IJDPS) Vol.3, No.2, March 2012

\begin{tabular}{|l|l|l|l|l|l|}
\hline MH3 & MH2 & 2 & S674_MH3 & T001_MH4 & Ptr1_MH3 \\
\hline MH4 & MH4 & 0 & S820_MH4 & T001_MH4 & Ptr1_MH4 \\
\hline MH5 & MH6 & 2 & S502_MH5 & T002_MH4 & Ptr1_MH5 \\
\hline MH6 & MH6 & 1 & S168_MH6 & T001_MH4 & Ptr1_MH6 \\
\hline MH7 & MH6 & 2 & S238_MH7 & T002_MH4 & Ptr1_MH7 \\
\hline MH8 & MH6 & 3 & S160_MH8 & T002_MH4 & Ptr1_MH8 \\
\hline
\end{tabular}

And the forwarding table will look like

Table 4. Forwarding table at MH4 after Movement of MH1

\begin{tabular}{|c|c|c|}
\hline Destination & Metric & Sequence Number \\
\hline MH1 & 3 & S516_MH1 \\
\hline MH2 & 1 & S238_MH2 \\
\hline MH3 & 2 & S674_MH3 \\
\hline MH4 & 0 & S820_MH4 \\
\hline MH5 & 2 & S502_MH5 \\
\hline MH6 & 1 & S186_MH6 \\
\hline MH7 & 2 & S238_MH7 \\
\hline MH8 & 3 & S160_MH8 \\
\hline
\end{tabular}

\subsubsection{Advantages of DSDV}

1. DSDV protocol guarantees loop free paths.

2. In DSDV count to infinity problem is reduced which was a major problem in Distance vector protocol.

3. Extra traffic can be avoided with incremental updates.

4. In routing table, DSDV not maintain multiple paths to destination. A good practice in DSDV is to maintain best paths to a destination only. Because of this space consumed by routing table is reduced.

\subsubsection{Limitations of DSDV}

1. Because of unnecessary advertisement of routing information bandwidth is wasted.

2. DSDV doesn't support Multi path Routing.

3. It is difficult to determine a time delay for the advertisement of routes .

4. For larger network it is difficult to maintain routing table..As all nodes in a network maintains table, it leads to overhead. which consumes more bandwidth.

5. Problem of fluctuation and damping fluctuation. 
International Journal of Distributed and Parallel Systems (IJDPS) Vol.3, No.2, March 2012

\section{Ad-hoc On-Demand Distance Vector (AODV) Protocol}

AODV protocol of MANET doesn't have a fixed topology in a network. This is basically needed for wireless communication for the nodes and links are created as and when required. The sequence number of routing table is used to determine whether the routing information is up-to-date or not and also it is useful to prevent routing loop problem. To routes are created on demand, source node broadcast (RREQ) request packet to their neighbours and neighbours relay the same until it reached to its destination. Then destination node sends reply packet to source node (RREP) using the same path from which request packet come.

\subsection{Working of AODV}

As previously stated, paths are formed as and when required in the network therefore, each node acts as a specialised router. The routing information also maintains two separate counters: a node sequence number and a broadcast-id. When a source node wants to communicate with destination, it increments its broadcast-id and initiates path discovery by broadcasting a route request packet RREQ to its neighbours. The RREQ contains the following fields:

1. source-addr

2. source-sequence

3. dest-addr

4. dest-sequence

5. hop-cnt

The RREQ packet is identify uniquely with the help of source-addr and broadcast id pair only. Later the dynamic route entries from source to destination for all nodes are maintained. The intermediate node updates routing information and propagates new RREP only,

- If the Destination sequence number is greater, or

- If the new sequence number is same and hop count is small, or Otherwise, it just skips the new RREP. This ensures that algorithm is loop-free and only the most effective route is used .[2]

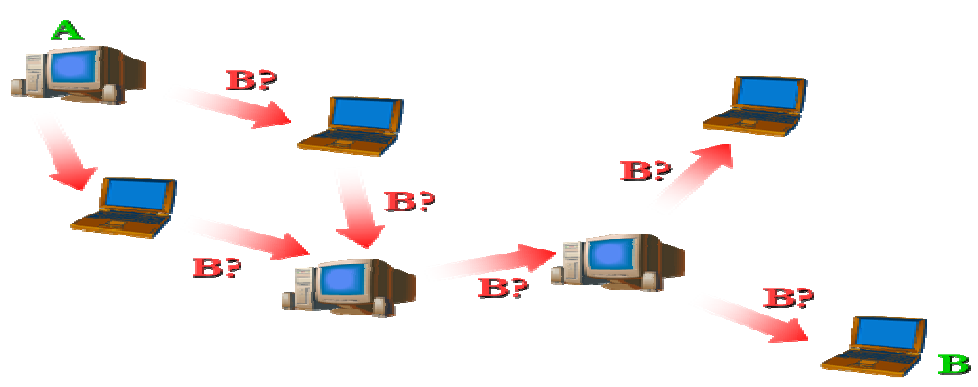

Figure 7. Propagation of RREQ

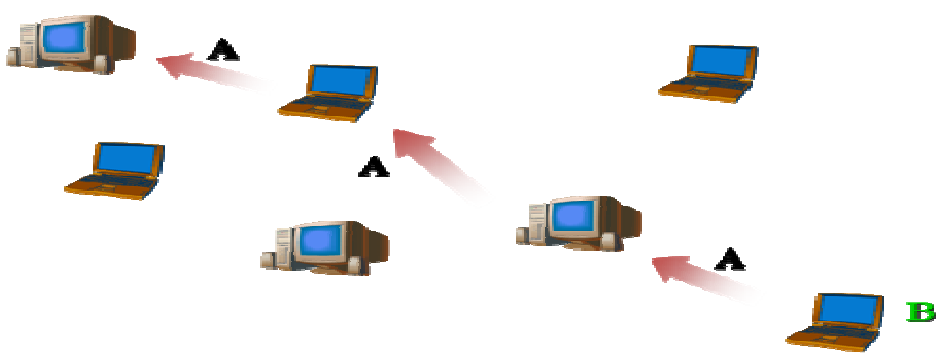

Figure 8. Propagation of RREP 
International Journal of Distributed and Parallel Systems (IJDPS) Vol.3, No.2, March 2012

\subsubsection{Route Table Management}

In the network, all nodes maintain routing table for each destination of interest in their routing table. Each entry contains the following info:

- Destination

- Next hop

- Number of hops

- Destination sequence number

- Active neighbours for this route

- Expiration time for the route table entry

Routing table contains the source and destination sequence number as well as it also maintains soft-state information which is associated with routing entries. To identify active source nodes along the path when the link to destination is breaks down this information is needed[6].

\subsection{Advantages of AODV}

1. AODV is suitable for VANET, because of its dynamic nature [1].

2. This protocol is basically used for unicast as well as multicasting of packets also..

\subsection{Limitations of AODV}

1. Requirement on broadcast medium: The algorithm expects/requires that the nodes in the broadcast medium can detect each others' broadcasts.

2. As the route is not initially known, so the request packet travels from node to other nodes in a network to find out route information on demand. It also maintains the address of all nodes through which it passing, so the reverse path formed. Therefore the overhead on bandwidth is occurred.

3. It lacks an efficient route maintenance technique; because the routing information is always obtained on demand which also contain common traffic information, which will may not be reusable.

4. AODV lacks support for high throughput routing metrics: AODV is designed to support the shortest hop count metric. This metric favours long, low bandwidth links over short, high-bandwidth links.

5. As this protocol is reactive in nature, so until the flow is initiated, it will not discover a route. Therefore route discovery latency is high for large networks like mesh.

\section{Comparison of AODV and DSDV}

Following review of two protocols i.e., AODV and DSDV is based on the above stated study.

1. Both protocol used the concept of sequence number to update latest routing information.

2. The bandwidth is wasted in case of DSDV, because of periodic broadcasting of updated information. In AODV, nodes only propagates hello messages to its neighbours.

3. In DSDV, routes information which are maintained in routing table can be stale as DSDV cannot handle nodes movement at high speeds due to lack of alternative routes.. But in case of AODV, the routes are find out on demand only, so the routes information can not be stale.

4. In DSDV, throughput decreases because of periodic routes information updates and if the node mobility is at high speed. In AODV, throughput is stable because it don't broadcast any routing information.

While comparing two protocols, we also focus on two performance measurements such as Average Delay, Packet Delivery Fraction. 
(i) Packet delivery fraction: The ratio of the number of data packets successfully delivered to the destinations to those generated by CBR sources. Packet delivery fraction $=$ (Received packets/Sent packets)*100.

(ii) Average End to end delay of data packets: The average time from the beginning of a packet transmission at a source node until packet delivery to a destination. This includes delays caused by buffering of data packets during route discovery, queuing at the interface queue, retransmission delays at the MAC, and propagation and transfer times. Calculate the send(S) time $(\mathrm{t})$ and receive $(\mathrm{R})$ time $(\mathrm{T})$ and average it.[2]

Table 5. Comparison between AODV and DSDV

\begin{tabular}{|l|l|l|}
\hline $\begin{array}{l}\text { Protocol } \\
\text { Parameter }\end{array}$ & $\begin{array}{l}\text { Reactive } \\
\text { AODV }\end{array}$ & $\begin{array}{l}\text { Proactive } \\
\text { DSDV }\end{array}$ \\
\hline Broadcasting & $\begin{array}{l}\text { Hello messages are } \\
\text { propagated to neighbour }\end{array}$ & Done periodically \\
\hline $\begin{array}{l}\text { Sending data to a particular } \\
\text { node }\end{array}$ & Has to find a route & $\begin{array}{l}\text { Maintains all the route in } \\
\text { routing table .No need to } \\
\text { find route. }\end{array}$ \\
\hline $\begin{array}{l}\text { End to End } \\
\text { Delay }\end{array}$ & Medium & High \\
\hline Routing Loop avoidance & Yes & Yes \\
\hline Flooding & Yes & Yes \\
\hline $\begin{array}{l}\text { Power } \\
\text { Consumption }\end{array}$ & Medium & High \\
\hline $\begin{array}{l}\text { Packet delivery In high } \\
\text { mobility }\end{array}$ & More & $\begin{array}{l}\text { Comparatively } \\
\text { Less }\end{array}$ \\
\hline
\end{tabular}

\section{Conclusion}

From the above analysis its being cleared that Distance Vector protocol actually not well suited for Ad hoc network therefore, DSDV protocol is discussed which overcomes problems of distance vector protocol. From the overall analysis, AODV protocol is better than DSDV, because DSDV consumes ore bandwidth as it periodically broadcast routing information, whereas in AODV there is no need to maintain route table, which results in less bandwidth consumption as well as less overhead. For small network, DSDV works well and AODV is best suited for larger network. Even the throughput is less in DSDV as it continuously broadcast route information, but in case of AODV throughput is stable as it don't needed to maintain any route information.

\section{References}

[1] Ahmed Al-Maashri and Mohamed Ould-Khaoua."Performance Analysis of MANET Routing Protocols in the Presence of Self-Similar Traffic". IEEE, ISSN- 0742-1303, First published in Proc. of the $31^{\text {st }}$ IEEE Conference on Local Computer Networks, 2006.

[2] C. E. Perkins, E. M. Royer, and S. R. Das, "Ad Hoc On- Demand Distance Vector (AODV) Routing”, Internet Draft, draft-ietf-manet aodv-10.txt, work in progress, 2002.

[3] J. Broch, D. A. Maltz, D. B. Johnson, Y-Ch Hu, and J. Jetcheva, "A Performance Comparison of Multi-Hop Wireless Ad Hoc Network for mobile ad hoc networks," in Proc. 4th annual 
International Journal of Distributed and Parallel Systems (IJDPS) Vol.3, No.2, March 2012

IEEE/ACM international conference on Mobile Computing and Networking, Dallas, Texas, U.S.Oct. 1998, pp. 85-97.

[4] Performance Comparison and Analysis of DSDV and AODV for MANET V.Ramesh, Dr.P.Subbaiah, N. Koteswar Rao, M.Janardhana Raju .

[5] Rima Khalaf Ali El-Haj-Mahmoud and Ayman Kayssi. Performance comparison of the aodv and dsdv routing protocols in mobile ad hoc networks. Technical report, Department of Electrical and Computer Engineering, American University of Beirut, USA.

.[6] R. S. Sisodia, B. S. Manoj and C. Siva Ram Murthy, "A Preferred Link- Based Routing Protocol for Ad Hoc Wireless Networks," Journal of Communications and Networks, vol. 4, no. 1, march 2002, pp. 14-21.

[7] S.Manga, A.Tamilarasi," Evaluation of the Performance Metrics in Improved Location Aided Cluster based Routing Protocol for GPS Enabled MANETs", European Journal of Scientific Research ISSN 1450-216X Vol.46 No.2 (2010), pp.296-308.

[8] Jiann-Liang Chen1, Yin-Fu Lai1, Hsi-Feng Lu1,2 and Quan-Cheng Kuo1, $\square$ Public-key Based Security Scheme for Wireless Sensor Network $\square$, in 2008 IEEE pp. 255-258

[9] Shi-Chun Tsai, Wen-Guey Tzeng, and Kun-Yi Zhou," Key Establishment Schemes against Storage-Bounded Adversaries in Wireless Sensor Networks", in IEEE TRANSACTIONS ON WIRELESS COMMUNICATIONS, VOL. 8, NO. 3, MARCH 2009, pp. 1218-1222. 